\title{
Presença de lactose em medicamentos isentos de prescrição
}

\author{
Presence of lactose in nonprescription drugs \\ Marcia Otto Barrientos ${ }^{1 *}$, Fernanda Cristina Figueira Teixeira², Roberto Paulo Correia de Araújo ${ }^{3}$ \\ ${ }^{1}$ Mestre em Ciências - CENA/USP, Farmacêutica, FAFABES/UFES. Professora da Faculdade Adventista da Bahia \\ - FADBA; ${ }^{2}$ Mestre em Ciências da Saúde pela UFU, Doutoranda pela UNICAMP; ${ }^{3}$ Professor Livre Docente de \\ Bioquímica e Permanente do Programa de Pós-graduação em Processos Interativos dos órgãos e Sistemas, UFBA.
}

\begin{abstract}
Resumo
Introdução: a polifarmácia e a automedicação estão presentes na população brasileira, sendo frequente sua ocorrência com medicamentos isentos de prescrição. Ademais, a lactose é utilizada como excipiente em formas farmacêuticas sólidas. Objetivo: o presente estudo constitui uma pesquisa sobre lactose, na condição de excipiente, em medicamentos isentos de prescrição (MIPs). Metodologia: em 391 MIPs presentes em cinco formas farmacêuticas sólidas e analisadas por classes, categorias e marcas, foram coletadas, nos meses de abril e maio de 2018, informações quanto à presença de lactose em bulas disponíveis nos sites da Agência Nacional de Vigilância Sanitária e da indústria farmacêutica no Brasil. Resultados: constatou-se que a lactose está presente em 43,48\% dos MIPs, sendo mais frequente nos comprimidos e comprimidos revestidos, nas proporções de $42,94 \%$ e $36,47 \%$, respectivamente. Ela foi encontrada, predominantemente, em medicamentos similares, principalmente antialérgicos, analgésicos não opioides e anti-inflamatórios não esteroides, bem como na totalidade dos antidiarreicos e laxantes. Das 88 marcas analisadas, 30 apresentaram mais de $50 \%$ dos produtos com lactose. Identificou-se um percentual expressivo de medicamentos utilizados no controle dos sintomas de intolerância à lactose que continham esse carboidrato como excipiente. Conclusão: nas formas farmacêuticas analisadas, a presença de lactose nos MIPs é superior a 40\%. A lactose presente pode ser agravante de sintomas gastrintestinais. Portanto, é essencial incluir, na embalagem dos medicamentos, a frase de alerta sobre a presença desse açúcar, visando à sua ampla divulgação, particularmente dirigida aos indivíduos com restrição de uso dessa substância.

Palavras-chave: Intolerância à lactose. Excipientes. Medicamentos sem prescrição.
\end{abstract}

\begin{abstract}
Introduction: polypharmacy and self-medication are present in Brazilian population, frequently occurring for prescription-free medicines. In addition, lactose is used as an excipient in solid dosage forms of prescription-free medicines. Objective: the present study constitutes a research on lactose, as an excipient, in prescription-free medicines (MIPs). Methodology: in 391 MIPs present in five solid dosage pharmaceutical forms and analyzed by classes, categories and brands, in April and May 2018, information was collected on the presence of lactose in the package inserts available on the websites of the National Sanitary Surveillance Agency and on the pharmaceutical industry websites in Brazil. Results: it was found that lactose is present in $43.48 \%$ of the MIPs, being more frequent in tablets and coated tablets, in the proportions of $42.94 \%$ and $36.47 \%$, respectively. It has been found predominantly in similar drugs, mainly antiallergics, non-opioid analgesics and non-steroidal anti-inflammatory drugs, as well as in all antidiarrheals and laxatives. Of the 88 brands analyzed, 30 presented over $50 \%$ of lactose products. An expressive percentage of drugs used to control the symptoms of lactose intolerance that contained this carbohydrate as an excipient were identified. Conclusion: in the pharmaceutical forms analyzed, the presence of lactose in the MIPs is higher than $40 \%$. The lactose present may be aggravating gastrointestinal symptoms. It is therefore essential to include a warning on the presence of this sugar on the packaging of medicinal products, with a view to their wide dissemination, particularly for those who have a restricted use of that substance.

Keywords: Lactose Intolerance. Excipients. Nonprescription Drugs.
\end{abstract}

\section{INTRODUÇÃO}

As formas farmacêuticas sólidas são práticas de administrar, apresentam dosagem precisa e excelente estabilidade. São apresentadas, principalmente, como sólidos aglomerados, destacando-se os comprimidos, as drágeas, as cápsulas, as pastilhas, as pílulas e os granulados, sendo o comprimido a forma mais abundante na indústria farmacêutica. A maioria dos princípios ativos não apresenta características mecânicas adequadas para

Correspondente/Corresponding: *Marcia Otto Barrientos - End: Faculdade Adventista da Bahia. BR $101 \mathrm{Km}$ 197, Cx. postal 18. 44300-000. Cachoeira, Ba-Tel: (75) 99137-5756-E-mail: mbarrientos22@gmail.com. sofrer compressão. ${ }^{(1,2)}$ Desse modo, na fabricação de medicamentos, ingredientes inativos, denominados de excipientes, são combinados e agregados intencionalmente aos princípios ativos, para dar a estabilidade adequada à sua forma farmacêutica. ${ }^{(3)}$

A introdução da lactose como excipiente revolucionou a forma de fabricação de comprimidos na década de 60, aumentando a resistência ao esmagamento, com a vantagem de possuir excelente estabilidade físico-química, sabor suave, disponibilidade, e ainda boa relação entre custo e benefício. ${ }^{(4)}$ Estudos recentes apontam que a lactose, coprocessada a outros excipientes, apresenta vantagens 
características, mantendo-se como uma substância dentre as primeiras escolhas para os excipientes sólidos. ${ }^{(4,5)}$

A legislação brasileira, RDC 47 de 08 de setembro de 2009, determina a descrição qualitativa dos excipientes na bula dos medicamentos e a inserção de frases de alerta no caso de precauções para o uso. ${ }^{(6)}$ As frases de alerta para princípios ativos e excipientes em bulas e rotulagem de medicamentos são regulamentadas pela RDC 60 , de 17 de dezembro de 2010, que não rege sobre a presença de lactose. ${ }^{(7)}$ Portanto, no Brasil, a quantidade de lactose por unidade posológica de medicamento não é apresentada e não há frase de alerta nas caixas indicando sua presença. Além disso, há de se considerar a prática da automedicação que, segundo Arrais et al. (2016), tem prevalência de 16,1\% na população brasileira, sendo que $65,5 \%$ das pessoas que se automedicam o fazem com medicamentos isentos de prescrição (MIPs). ${ }^{(8)}$ Os critérios para o enquadramento de um fármaco como isento de prescrição estão na RDC no 98 de 01/08/2016. ${ }^{(9)}$ Tais critérios incluem: apresentar baixo potencial de risco; não gerar dependência; ser manejável pelo paciente; ser indicado para sintomas identificáveis e ser seguro. Incluem ainda o tempo de utilização total do medicamento e sua comercialização no mercado internacional. A Instrução Normativa no 11 , de 29 de setembro de 2016 apresenta a lista de medicamentos isentos de prescrição (LMIP), categorizada por grupos terapêuticos, com as respectivas indicações, limitando, através das observações, os produtos com restrição ou os permitidos para cada categoria. ${ }^{(10)}$

A adição de lactose aos medicamentos e a não regulamentação da presença de uma frase de alerta nas caixas ganham relevância, principalmente, frente à possibilidade de consumo de tais medicamentos por indivíduos intolerantes à lactose, devido à hipolactasia, que é a diminuição da ação enzimática da lactase, mais comum em adultos. A hipolactasia primária é decorrente de uma expressão gênica que reduz a formação da enzima ao longo da vida. A hipolactasia secundária pode ser reversível, dependendo da doença que provoque a redução da ação enzimática. A forma congênita é rara e pode ser fatal, se não for diagnosticada aos primeiros sintomas. As consequências da hipolactasia primária e secundária são a má absorção e a intolerância à lactose. A má absorção ocorre quando há hipolactasia parcial, sem a presença de sintomas regulares; já a intolerância à lactose constitui a presença de sintomas decorrentes da fermentação da lactose pela microflora intestinal. ${ }^{(11,12)}$

A prevalência de hipolactasia primária no adulto difere em diferentes populações. No Brasil, brancos e pardos apresentam $57 \%$ de incidência, que é superior a $80 \%$ em índios e negros, alcançando $100 \%$ em pessoas de descendência japonesa. ${ }^{(11)} A$ variedade e a intensidade dos sintomas, na intolerância à lactose, dependem de fatores intrínsecos ao paciente e a seus hábitos; por isso, não há categorização com um protocolo de quantidade de lactose a ser ingerida por cada paciente, sendo a restrição, frequentemente, indicada como parte essencial no tratamento. ${ }^{(12)}$
Constatar a presença de lactose como excipiente em medicamentos sólidos, isentos de prescrição, disponíveis no mercado brasileiro, foi o que motivou o presente estudo.

\section{METODOLOGIA}

Este é um estudo descritivo, com abordagem quantitativa, realizado nos meses de abril e maio de 2018. A classificação dos MIPs e a escolha dos princípios ativos resultaram dos dispositivos disciplinados pela Instrução Normativa, IN no 11, de 29 de setembro de 2016. ${ }^{(10)}$ A seleção dos medicamentos com as respectivas formas farmacêuticas foi realizada mediante a análise da lista de conformidade de preços disponibilizada pela Câmara de Regulação de Mercado de Medicamentos (CMED), atualizada em 9 de abril de 2018, considerada a ordem alfabética dos princípios ativos. ${ }^{(13)}$

Foram selecionados 391 medicamentos de 88 marcas, agrupados de acordo com as formas farmacêuticas, classes de MIPs e categorias. As formas farmacêuticas analisadas foram: comprimidos, comprimidos revestidos, drágeas, cápsulas e cápsulas duras de gel. As classes incluíram: analgésicos não opioides, aminoácidos, vitaminas e minerais (AAVM), anti-inflamatórios não esteroides, antialérgicos, antigripais, antiflatulentos, relaxantes musculares, antiespasmódicos, antidiarreicos, antiparasitários, laxantes, colagogos, antissépticos urinários e tônicos orais. As categorias incluíram: medicamentos de referência, genéricos, similares, específicos, fitoterápicos e biológicos.

Os excipientes indicados nos prospectos profissionais disponibilizados no bulário eletrônico ${ }^{(14)}$ da Agência Nacional de Vigilância Sanitária (ANVISA) e nos sites da indústria farmacêutica foram cuidadosamente analisados. As categorias dos medicamentos avaliados baseiam-se nas listas de referência A e B, disponibilizadas em 12 de abril de $2018^{(15)}$ na lista de medicamentos genéricos registrados, atualizada até o Diário Oficial da União de 30 de junho de $2017^{(16)}$, na lista de medicamentos similares e seus respectivos medicamentos de referência, conforme RDC 58/2014 atualizada até 28 de fevereiro de $2018^{(17)}$, segundo o Diário Oficial da União, e a 1a edição do Memento Fitoterápico da Farmacopeia Brasileira. ${ }^{(18)}$

Para medicamentos com concentrações diferentes de um mesmo fármaco numa mesma forma farmacêutica e de uma mesma marca, apenas uma apresentação foi selecionada. Havendo excipientes diferentes entre concentrações ou formas farmacêuticas de um mesmo fármaco, ambos foram considerados.

\section{RESULTADOS}

Dos 391 medicamentos selecionados para estudo, $170(43,48 \%)$ continham lactose nos excipientes. A Tabela 1 apresenta a distribuição dos medicamentos, de acordo com a forma farmacêutica. Entre todos os medicamentos que continham lactose, os comprimidos foram os mais frequentes $(42,94 \%)$, seguidos dos comprimidos revestidos $(36,47 \%)$. Ao se considerar a frequência dentro de cada 
forma farmacêutica, constatou-se que mais de $70 \%$ das cápsulas e mais de metade das drágeas continham lactose.

Tabela 1 - Presença de lactose de acordo com a forma farmacêutica dos MIPs. Brasil, maio de 2018.

\begin{tabular}{lrcc}
\hline Forma farmacêutica & $\mathbf{n}$ & $\begin{array}{c}\text { Presença de lactose } \\
\mathbf{n}(\%)\end{array}$ & $\begin{array}{c}\text { Proporção* } \\
\text { (\%) }\end{array}$ \\
\hline Comprimido & 185 & $73(39,46)$ & 42,94 \\
Comprimido revestido & 154 & $62(40,26)$ & 36,47 \\
Cápsula dura de gel & 20 & $15(75,00)$ & 8,82 \\
Drágea & 21 & $12(57,14)$ & 7,06 \\
Cápsula & 11 & $8(72,73)$ & 4,71 \\
TOTAL & 391 & $170(43,48)$ & 100,00 \\
\hline
\end{tabular}

* Proporção calculada com relação ao total de MIPs com lactose (n $=170$ )

MIPs - medicamentos isentos de prescrição

Fonte: Autoria própria.

Nas classes de MIPs analisadas, foi verificado que todos os antidiarreicos e laxantes continham lactose nos excipientes, o que ocorreu também com mais da metade dos anti-inflamatórios, antialérgicos, antiflatulentos, antiespasmódicos, antiparasitários e colagogos. A ausência da lactose foi verificada em todos os tônicos orais (Tabela 2). Nos medicamentos com lactose nos excipientes, os aminoácidos, vitaminas e minerais (AAVM) tiveram a maior proporção (20,59\%); os analgésicos não opioides, os anti-inflamatórios não esteroides e os antialérgicos contribuíram com frequências superiores a $10 \%$.

Tabela 2 - Presença de lactose de acordo com a classe dos MIPs. Brasil, maio de 2018.

\begin{tabular}{lrrc}
\hline Classe & n & $\begin{array}{c}\text { Presença de lactose } \\
\mathbf{n}(\%)\end{array}$ & $\begin{array}{c}\text { Proporção* } \\
\text { (\%) }\end{array}$ \\
\hline Analgésicos NOP & 93 & $17(18,28)$ & 10,00 \\
AAVM & 91 & $35(38,46)$ & 20,59 \\
Anti-inflamatórios NE & 43 & $26(60,47)$ & 15,29 \\
Antialérgicos & 36 & $22(61,11)$ & 12,95 \\
Antigripais & 30 & $11(36,67)$ & 6,47 \\
Antiflatulentos & 20 & $11(55,00)$ & 6,47 \\
Relaxantes musculares & 19 & $4(21,05)$ & 2,35 \\
Antiespasmódicos & 17 & $10(58,82)$ & 5,88 \\
Antidiarreicos & 15 & $15(100,00)$ & 8,82 \\
Antiparasitários & 11 & $9(81,82)$ & 5,29 \\
Laxantes & 7 & $7(100,00)$ & 4,12 \\
Colagogos & 3 & $2(66,67)$ & 1,18 \\
Antissépticos urinários & 3 & $1(33,33)$ & 0,59 \\
Tônicos orais & 3 & $0(-)$ & - \\
TOTAL & 391 & $170(43,48)$ & 100,00 \\
\hline
\end{tabular}

*Proporção calculada com relação ao total de MIPs com lactose ( $\mathrm{n}$ $=170$ ).

AAVM - aminoácidos, vitaminas e minerais; MIPs - medicamentos isentos de prescrição; NE - não esteroides; NOP - não opioides. Fonte: Autoria própria.
Considerando as categorias de medicamentos analisadas, a maior ocorrência de lactose foi verificada nos medicamentos similares (44,12\%), seguida dos específicos (20,59\%). Todos os medicamentos biológicos analisados continham lactose, assim como mais da metade dos fitoterápicos e metade dos MIPs de referência (Tabela 3).

Tabela 3 - Presença de lactose de acordo com a categoria dos MIPs. Brasil, maio de 2018.

\begin{tabular}{llcc}
\hline Categoria & $\mathrm{n}$ & $\begin{array}{c}\text { Presença de lactose } \\
\mathrm{n}(\%)\end{array}$ & $\begin{array}{c}\text { Proporção* } \\
(\%)\end{array}$ \\
\hline Biológico $^{1}$ & 10 & $10(100,00)$ & 5,88 \\
Específico $^{2}$ & 91 & $35(38,46)$ & 20,59 \\
Fitoterápico $^{3}$ & 3 & $2(66,67)$ & 1,18 \\
Genérico $^{4}$ & 73 & $28(38,36)$ & 16,47 \\
Referência $^{5}$ & 40 & $20(50,00)$ & 11,76 \\
Similar $^{6}$ & 174 & $75(43,10)$ & 44,12 \\
TOTAL & 391 & $170(43,48)$ & 100,00 \\
\hline
\end{tabular}

1 - Probiótico.

2 - Aminoácidos, vitaminas e minerais segundo as RDC $24 / 2011^{(19)}$ e RDC 97/2016 (20).

3 - Exclusivamente de matérias primas vegetais

4 - Intercambiável ao medicamento de referência, identificado pelos princípios ativos.

5 - Produto inovador, com eficácia, segurança e qualidade aprovadas. 6 - Equivalente ao medicamento de referência, identificado por nome comercial.

*Proporção calculada com relação ao total de MIPs com lactose (n $=170$ ).

MIPs - medicamentos isentos de prescrição.

Fonte: Autoria própria.

As classes de MIPs foram relacionadas com as categorias de medicamentos biológicos, genéricos, referência e similares. As frequências de presença e ausência de lactose são apresentadas na Tabela 4. Nessa relação, considerando o total dos MIPs avaliados, a lactose foi encontrada com maior frequência nos antialérgicos similares $(3,07 \%)$. As proporções dos analgésicos não opioides e anti-inflamatórios não esteroides, ambos similares e contendo lactose, foram semelhantes na amostra $(2,81 \%)$, o mesmo ocorrendo com os antigripais similares e antidiarreicos biológicos (2,56\%). Nas categorias de medicamentos genéricos e nos de referência, os anti-inflamatórios não esteroides apresentaram maior frequência de lactose, $2,30 \%$ e $1,53 \%$, respectivamente. Dentre os analgésicos não opioides, a lactose foi encontrada com maior frequência no fármaco paracetamol $(49,46 \%)$, seguindo-se de dipirona (29,03\%). Todos os colagogos estudados são fitoterápicos, e neles foram encontrados dois medicamentos com lactose e um sem lactose. 
Tabela 4 - Distribuição das classes dos MIPs por categorias de medicamentos, de acordo com a presença ou ausência de lactose. Brasil, 2018.

\begin{tabular}{lccccccccc}
\hline & \multicolumn{1}{c}{ Categorias } & \multicolumn{2}{c}{1} & \multicolumn{2}{c}{2} & \multicolumn{3}{c}{3} & \multicolumn{2}{c}{4} \\
Classes & Sim & Não & Sim & Não & Sim & Não & Sim & Não \\
\hline Analgésicos NOP & - & - & 5 & 34 & 1 & 8 & 11 & 34 \\
Anti-inflamatórios NE & - & - & 9 & 2 & 6 & 1 & 11 & 14 \\
Antialérgicos & - & - & 7 & 5 & 3 & 1 & 12 & 8 \\
Antidiarreicos & 10 & 0 & - & - & 1 & 0 & 4 & 0 \\
Antiespasmódicos & - & - & - & - & 3 & 1 & 7 & 6 \\
Antigripais & - & - & 0 & 1 & 1 & 4 & 10 & 14 \\
Antiflatulentos & - & - & 6 & 3 & 1 & 1 & 4 & 5 \\
Antiparasitários & - & - & 1 & 0 & 3 & 0 & 5 & 2 \\
Antissépticos urinários & - & - & - & - & - & - & 1 & 2 \\
Laxantes & - & - & - & - & 1 & 0 & 6 & 0 \\
Relaxantes musculares & - & - & - & - & 0 & 3 & 4 & 12 \\
Tônicos orais & - & - & - & - & 0 & 1 & 0 & 2 \\
\hline
\end{tabular}

1 - Biológicos - Probióticos.

2 - Genéricos - Intercambiáveis ao medicamento de referência, identificados pelos princípios ativos.

3 - Referências - Produtos inovadores, com eficácia, segurança e qualidade aprovadas.

4 - Similares - Equivalentes ao medicamento de referência, identificados por nomes comerciais.

MIPs - medicamentos isentos de prescrição; NOP - não opioides; $\mathrm{NE}$ - não esteroide.

Fonte: Autoria própria.

Em se tratando das marcas dos medicamentos, foi observado que, das 88 marcas, 32 estiveram representadas com apenas um medicamento; dessas, 38,71\% continham lactose. Das 56 marcas que abrangiam entre 2 e 24 medicamentos, 30 marcas apresentaram lactose em mais de $50 \%$ dos medicamentos, sendo que três delas apresentaram lactose em todos os medicamentos. Cabe ressaltar que uma dessas marcas pertence a um grupo farmacêutico brasileiro. A ausência de lactose foi observada em todos os medicamentos de oito marcas, incluindo cinco brasileiras.

Sobre a forma de associação da lactose, foi observado que, em 12 medicamentos, estavam presentes as associações de lactose com esteareato de magnésio e, em todos os demais, a associação ocorria com, no mínimo, uma das seguintes substâncias: celulose microcristalina, croscarmelose de sódio, amido e polivinilpirrolidona (PVP) e seus derivados.

\section{DISCUSSÃO}

Embora a preocupação com o uso e efeitos dos MIPs seja uma questão internacionalmente discutida ${ }^{(20,21)} \mathrm{e}$ investigada, há poucos estudos acerca da participação dos excipientes a eles adicionados, em particular a lactose. ${ }^{(22,23)} \mathrm{Em}$ 2008, uma análise do Physician's Desk Reference
(Itália), registrou que $6 \%$ dos medicamentos de livre comercialização e $33 \%$ dos medicamentos orais continham lactose como excipiente. ${ }^{(24)}$

O presente estudo apontou que quase metade dos MIPs analisados contém lactose. Essa substância está presente, em maior proporção, na forma de comprimidos, na categoria dos similares e na classe dos aminoácidos, vitaminas e minerais. As frequências foram maiores do que as apontadas por Stefani et al. ${ }^{(25)}$, quando investigaram a presença de lactose em 181 medicamentos habitualmente prescritos por pediatras no Brasil, em 11 formas farmacêuticas e 11 classes diferentes, tendo concluído que $28 \%$ continham lactose. Os valores inferiores daquele estudo se devem, possivelmente, à abrangência de todas as formas farmacêuticas orais, não se considerando a função da lactose como excipiente. Por suas características e seu histórico, os principais estudos da funcionalidade da lactose em sólidos são desenvolvidos em comprimidos. ${ }^{(4)}$ $O$ presente estudo informa que a presença de lactose entre comprimidos e comprimidos revestidos foi de $79,41 \%$ nos MIPs analisados. Os excipientes diferem entre comprimidos e comprimidos revestidos, pois esses últimos necessitam de condições apropriadas para se evitarem defeitos de adesão, falta de uniformidade de cor e brilho, bolhas e rachaduras. ${ }^{(26)}$

A dipirona, associada ou não, foi apontada como o medicamento de maior consumo por automedicação no Brasil. ${ }^{\left({ }^{8}\right)}$ Esse achado é discordante da disponibilidade de apresentações de analgésicos sólidos no mercado brasileiro. Observou-se que, dentre os 93 analgésicos analisados, 28 estão em associação com a cafeína, e o fármaco analgésico mais disponível foi o paracetamol (n = 46). Quanto à presença de lactose em excipientes de analgésicos, o estudo de Stefani et al. ${ }^{(25)}$ revelou a ausência de lactose em sete marcas de analgésicos em comprimidos; dessas, quatro que participaram do presente estudo apresentaram resultados concordantes. Em um estudo brasileiro sobre excipientes com potencial de induzir reações adversas ${ }^{(23)}$, a presença de lactose foi constatada em uma formulação de dipirona associada ao butilbrometo de escopolamina. Essa mesma associação de fármacos, pertencente à classe de antiespasmódicos, foi avaliada no presente estudo, revelando um percentual da ordem de $63,64 \%$ dos antiespasmódicos que continham lactose.

Há mais de 700 drogas que podem provocar a diarreia. Dentre as drogas referidas pelo estudo de Chassany, Mixeaux e Bergmann ${ }^{(27)}$, a lactose é apontada como desencadeadora desse efeito intestinal. Admite-se que a presença de lactose em medicamentos empregados no tratamento de distúrbios gastrintestinais pode contribuir para o surgimento ou manutenção desses sintomas. ${ }^{(28)}$ No presente estudo, $100 \%$ dos medicamentos antidiarreicos analisados - nos quais se enquadram também os repositores de flora intestinal e $52,38 \%$ dos antiflatulentos - apresentam lactose nos excipientes. Mais de $60 \%$ dos anti-inflamatórios e antialérgicos avaliados também contêm lactose, o que está em conformidade com estudos ${ }^{(25)}$ 
que identificaram a ocorrência expressiva desse açúcar nessas classes de MIPs. Contraditoriamente, dentre os sintomas da intolerância à lactose, estão a presença de diarreia ocasional ou crônica, com a ocorrência de gases intestinais, e a rinite alérgica ${ }^{(12)}$, havendo até a possibilidade de o indivíduo ingerir o agente agressor na tentativa de tratar seus efeitos.

Não há dose mínima de ingestão de lactose estabelecida para que se desenvolvam os sintomas de intolerância. Estudo realizado com 20 indivíduos constatou que 75\% deles apresentaram os sintomas com $12 \mathrm{~g}$ de lactose ou até menos, enquanto que, no teste de intubação intestinal, foi detectado um aumento de líquido e sódio no intestino delgado com apenas $3 \mathrm{~g}$ de lactose. ${ }^{(29)} \mathrm{Em}$ contrapartida, estudo comparativo realizado com 30 pessoas autorreferidas como severamente intolerantes à lactose e confirmadas com o teste de hidrogênio expirado após a ingestão de $15 \mathrm{~g}$ de lactose concluiu que não houve diferenças significativas entre grupos no que se refere aos sintomas intestinais após a ingestão de um copo de leite por dia, ou seja, o equivalente a $12 \mathrm{~g}$ de lactose. ${ }^{(30)}$

Considerando que cada forma farmacêutica sólida não tenha mais do que $400 \mathrm{mg}$ de lactose por unidade, Montalto et al. ${ }^{(24)}$ submeteram 77 pessoas com má digestão de lactose ao teste de hidrogênio expirado após a ingestão de uma cápsula de $400 \mathrm{mg}$ de lactose e compararam ao grupo placebo, não tendo encontrando diferença significativa na excreção de hidrogênio através da respiração e nem nos sintomas gastrointestinais. Estudos dessa natureza indicam um forte argumento para a recusa à inclusão de uma frase de alerta sobre a presença de lactose nos medicamentos. Em determinados países, algumas empresas tomam a iniciativa de alertar sobre a presença de lactose nas embalagens dos medicamentos $^{(30)}$. No Brasil, esse alerta está regulamentado para alimentos nas RDC $135 / 2017^{(31)}$ e RDC e 136/2017 $7^{(32)}$, que estabelecem a obrigatoriedade da frase "Contém lactose" nas embalagens de produtos com mais de $100 \mathrm{mg}$ de lactose para cada $100 \mathrm{~g}$ ou $100 \mathrm{~mL}$ do produto. A quantidade mínima de lactose referida é de $0,1 \%$ do alimento, sendo que essa porcentagem é inferior à quantidade de lactose presente por unidade posológica dos medicamentos. ${ }^{(24)}$

Devem-se considerar situações em que a presença da frase de alerta respeita o direito do consumidor e evita o agravamento de sintomatologias oriundas da baixa absorção da lactose. Há pessoas extremamente sensíveis à lactose como as que apresentam hipolactasia secundária decorrente de cirurgia ou de doença que reduz as vilosidades intestinais. Existem indivíduos hipersensíveis que fazem o uso restritivo ou evitam o consumo de muitas substâncias por não dominarem qual será o gatilho para a próxima crise alérgica. Há os que não consomem produtos de origem animal por opção ética, alimentar ou religiosa. Há de se considerar ainda as pessoas idosas, que possuem menor capacidade absortiva da lactose ${ }^{(33)}$, sendo um grupo que, por suas comorbidades, está sujeito à polifarmácia, que é o consumo de 5 ou mais medicamentos por dia, podendo chegar aos extremos de mais de 25 medicamentos diariamente. Se fosse possível somar a quantidade de lactose presente em todos esses medicamentos, haveria a possibilidade de serem alcançadas quantidades significativas dessa substância para o desenvolvimento de sintomas intestinais. ${ }^{(34,35)}$ Adicionalmente, a lactose presente nos excipientes de medicamentos soma-se à quantidade de lactose ingerida na alimentação diária. Esse fato, para uma pessoa que está regulando a ingestão diária de lactose, pode ser o diferencial entre a presença ou a ausência de sintomas gastrintestinais.

O presente estudo encontrou limitações no que se refere à obtenção de informações acerca de determinados medicamentos. $\mathrm{Na}$ categoria de genéricos, não foram encontradas as bulas de alguns princípios ativos nas formas farmacêuticas estudadas. Como exemplo, o butilbrometo de escopolamina não foi encontrado em comprimidos, apenas em gotas, sendo que ele se encontra em comprimidos nas categorias referência e similar. Outras categorias em que não foram encontradas bulas de genéricos isentos de prescrição nas formas analisadas foram os antidiarreicos, colagogos, laxantes, relaxantes musculares e antissépticos urinários. Foi observado que, entre os analgésicos não opioides, quatro marcas brasileiras apresentaram a presença dos mesmos excipientes em dois medicamentos, sendo um genérico e outro similar. Um grupo farmacêutico apresentou duas marcas de dipirona sódica $500 \mathrm{mg}$ genérica com os mesmos excipientes; entretanto, não foram encontradas as mesmas composições de excipientes em princípios ativos distintos. A ANVISA informa que há 3.870 medicamentos genéricos com registros válidos, dos quais $69,3 \%$ são de uso por via oral. Os medicamentos genéricos são produzidos após a expiração da patente, de acordo com a viabilidade de mercado analisada pelo fabricante. O Brasil fabrica $85,6 \%$ da totalidade de genéricos para o mercado interno, seguindo-se a Índia $(8,3 \%)$ e a Alemanha $(1,1 \%) .{ }^{(36)}$

Comparando-se a presença de lactose entre as marcas, foram encontradas evidências de que a presença de lactose talvez seja determinada, exclusivamente, pelas indústrias que, provavelmente, consideram fatores físico-químicos e econômicos na escolha dos excipientes. $\mathrm{Na}$ maioria dos casos, houve medicamentos com excipientes alternativos à presença de lactose para um mesmo fármaco. A lactose não é sugestiva de qualidade inferior, pois $50 \%$ dos medicamentos de referência a contém, embora seja sugestiva de baixo custo, pois medicamentos de menor preço, como os fármacos mebendazol, e cloridrato de loperamida, apresentaram marcante presença desse excipiente.

Não se pode pensar na lactose como um excipiente inerte. ${ }^{(37)}$ Apesar de possuir baixa reatividade, essa substância é um carboidrato redutor ${ }^{(38)}$ que, no estado sólido, é preparada em diferentes formas cristalinas. A $\alpha$-lactose mono-hidratada é um ligante relativamente fraco que, quando associado à celulose microcristalina, aumenta a resistência ao esmagamento. Para melhorar as carac- 
terísticas aglutinantes e desagregantes, associa-se esse polimorfo da lactose a substâncias como croscarmelose de sódio, amido, PVP e seus derivados. Neste estudo, apenas $7,06 \%$ dos medicamentos com presença de lactose não possuíam, pelo menos, uma dessas associações. Esses medicamentos se apresentam em cápsulas e cápsulas duras de gel e estão associados ao estearato de magnésio, um lubrificante para facilitar o escoamento do pó para dentro das cápsulas. Os polimorfos anidros possuem capacidades superiores de ligação, sendo que a forma isomérica $\alpha$ apresenta como desvantagem a lenta desintegração. Os demais polimorfos possuem propriedades de ligação superiores, sendo necessária a presença de uma substância desintegrante. . $^{(4,5,37)}$

Eadala et al. ${ }^{(28)}$ investigaram os medicamentos listados no British National Formulary (BNF) quanto à presença de lactose, concluindo que há dificuldade de o profissional médico identificar tal informação, por não haver referência a ela no BNF, sendo igualmente difícil para o paciente identificá-la, por não haver identificação na embalagem. (28) O mesmo ocorre com os pacientes no Brasil, que, na aquisição do medicamento, não conseguem acesso às bulas, porque as caixas estão lacradas, e os blisters de comprimidos vendidos avulsos geralmente não são acompanhados das respectivas bulas. Mesmo com a verificação dos excipientes na bula, o paciente pode não localizar a presença de lactose, pois ela pode estar em associações patenteadas, como o Ludipress $^{\circledR}$, o Microcelac ${ }^{\circledR}$, o Starlac ${ }^{\circledR}$ e o Cellactose ${ }^{\circledR(38)}$.

Este estudo avaliou a presença de lactose nos medicamentos sólidos isentos de prescrição. Sugere-se ampliar a investigação para uma amostra com maior diversidade de medicamentos orais, incluindo aqueles com venda sob a prescrição médica. Mediante a prevalência de hipolactasia primária no Brasil, bem como a existência da hipolactasia congênita e secundária ${ }^{(11,12)}$, considera-se essencial a regulamentação para a inclusão de frases de alerta nos rótulos dos medicamentos.

\section{CONCLUSÃO}

Com base nos achados do presente estudo, pode-se concluir: 1) a presença de lactose nos MIPs nas formas farmacêuticas analisadas é superior a $40 \%$, havendo maior ocorrência em comprimidos e comprimidos revestidos; 2 ) a quantidade de lactose presente nos medicamentos pode ser agravante de sintomas gastrintestinais em algumas situações específicas, até mesmo com medicamentos para os sintomas de intolerância à lactose que contêm lactose; 3) a inclusão de lactose como excipiente é decidida pela indústria farmacêutica, uma vez que, para um mesmo princípio ativo, há diferentes excipientes entre as marcas; 4 ) a melhor conduta será a inclusão de frases de advertência nas embalagens dos medicamentos que contenham lactose.

\section{AGRADECIMENTOS}

À Faculdade Adventista da Bahia (FADBA) pela assistência técnica e financiamento

\section{REFERÊNCIAS}

1. MIRANI, A. G. et al. Direct compression high functionality excipiente using coprocessing technique: a brief review. Curr. Drug Deliv., Beijing, v. 8 , n. 4, p. 426-435, 2011.

2. ROJAS, J.; BUCKNER, I.; KUMAR, V. Co-proccessed excipientes whitenhanced direct compression functionality for improved tableting performance. Drug Dev. Ind. Pharm., Abingdon, v. 38, n. 10, p. 11591170, 2012.

3. BRASIL. Agência Nacional de Vigilância Sanitária (ANVISA). Resolução RDC $n^{\circ} 34$, de 7 de agosto de 2015. Dispõe sobre as Boas Práticas de Fabricação de Excipientes Farmacêuticos. Diário Oficial [da] República Federativa do Brasil, Brasília, DF, 10 ago. 2015. Seção 1, p.41. Disponível em: <http://pesquisa.in.gov.br/imprensa/jsp/visualiza/index.jsp?jornal $=1 \&$ pagina=41\&data=10/08/2015>. Acesso em: 15 maio 2018.

4. GOHEL, M. C.; JOGANI, P. D. A review of co-processed directly compressive excipents. J. Pharm. Pharm. Sci., Alberta, v. 8, p. 76-93, 2005.

5. WANG, S. et al. Novel coprocessed excipientes composed of lactose, HPMC, and PVPP for tableting and its application. Int. J. Pharm., Amsterdam, v. 486, n. 1-2, p. 370-379, 2015.

6. BRASIL. Agência Nacional de Vigilância Sanitária (ANVISA). Resolução $\mathrm{RDC}^{\circ} 47$, de 08 de setembro de 2009. Estabelece regras para elaboração, harmonização, atualização, publicação e disponibilização de bulas de medicamentos para pacientes e para profissionais de saúde. Diário Oficial [da] República Federativa do Brasil, Brasília, DF, 09 set. 2009. Seção 1, p.31. Disponível em: <http://pesquisa.in.gov.br/imprensa/jsp/ visualiza/index.jsp?data=09/09/2009\&jornal=1\&pagina=31\&totalArqu ivos=80>. Acesso em: 16 maio 2018.

7. BRASIL. Agência Nacional de Vigilância Sanitária (ANVISA). Resolução $\mathrm{RDC}^{\circ}$ 60, de 17 de dezembro de 2010 . Estabelece frases de alerta para princípios ativos e excipientes em bulas e rotulagem de medicamentos. Diário Oficial [da] República Federativa do Brasil, Brasília, DF, 22 dez. 2010. Disponível em: < http://pesquisa.in.gov.br/imprensa/jsp/visualiza /index.jsp?jornal= $1 \&$ pagina $=83 \&$ data $=22 / 12 / 2010>$. Acesso em: 16 maio 2018.

8. ARRAIS, P. S. D. et al. Prevalence of self-medication in Braziland associated factors. Rev. Saúde Pública, São Paulo, v. 50, supl. 2, 13s, 2016. DOI:10.1590/S1518-8787.2016050006117.

9. BRASIL. Agência Nacional de Vigilância Sanitária (ANVISA). Resolução RDC n 98, de 01 de agosto de 2016. Estabelece os requisitos para declaração obrigatória da presença de lactose nos rótulos dos alimentos. Diário Oficial [da] República Federativa do Brasil, Brasília, DF, 03 ago. 2016. Disponível em: < http://pesquisa.in.gov.br/imprensa/jsp/ visualiza/index.jsp?jornal=1\&pagina=32 \&data=03/08/2016>. Acesso em: 03 abr. 2018.

10. BRASIL. Agência Nacional de Vigilância Sanitária (ANVISA). Instrução Normativa - IN n 11, de 29 de setembro de 2016. Dispõe sobre a lista de medicamentos isentos de prescrição. Diário Oficial [da] República Federativa do Brasil, Brasília, DF, 30 set. 2016. Seção1, p.99. Disponível em: <http://pesquisa.in.gov.br/imprensa/jsp/visualiza/index.jsp?jornal =1\&pagina=99\&data=30/09/2016>. Acesso em: 03 abr. 2018.

11. MATTAR, R.; MAZO, D. F. C. Intolerância à lactose: mudança de paradigmas com a biologia molecular. Rev. Assoc. Med. Bras., São Paulo, v. 56, n. 2, p. 230-236, 2010.

12. HURDUC, V. et al. Lactose intolerance: new aspects of an old Problem. Arch. Dis. Child. London, v. 102, supl. 2, p. A1-A192, 2017. 
13. AGÊNCIA NACIONAL DE VIGILÂNCIA SANITÁRIA (ANVISA). CÂMARA DE REGULAÇÃO DO MERCADO DE MEDICAMENTOS (CMED). Preços máximos de medicamentos por princípio ativo. jan. 2018. Disponível em: < http://portal.anvisa.gov.br/documents/374947/2829072/ LISTA+CONFORMIDADE_2018-01-26.pdf/21a9b762-f2df-4715-bbb7a588ddb9642d>. Acesso em: 03 abr. 2018.

14. AGÊNCIA NACIONAL DE VIGILÂNCIA SANITÁRIA (ANVISA). Bulário eletrônico. Disponível em: <http://www.anvisa.gov.br/datavisa/ fila_bula/index.asp>. Acesso em: 03 abr. 2018.

15. AGÊNCIA NACIONAL DE VIGILÂNCIA SANITÁRIA (ANVISA). Listas A e $B$ de medicamentos de referência. 2018. Disponível em: $<$ http://portal. anvisa.gov.br/registros-e-autorizacoes/ medicamentos/produtos/ medicamentos-de-referencia/lista>. Acesso em: 15 abr. 2018.

16. AGÊNCIA NACIONAL DE VIGILÂNCIA SANITÁRIA (ANVISA). Lista de medicamentos genéricos registrados. jun. 2017. Disponível em: < http://portal.anvisa. gov.br/ medicamentos-genericos-registrados>. Acesso em: 12 abr. 2018.

17 AGÊNCIA NACIONAL DE VIGILÂNCIA SANITÁRIA (ANVISA). Lista de medicamentos similares e seus respectivos medicamentos de referência, conforme RDC 58/2014. fev. 2018. Disponível em: < http://portal.anvisa. gov.br/medicamentos-similares>. Acesso em: 12 abr. 2018.

18. AGÊNCIA NACIONAL DE VIGILÂNCIA SANITÁRIA (ANVISA). Memento Fitoterápico Farmacopéia Brasileira. Brasília, 2016. Disponível em: < http://portal.anvisa.gov.br/documents/33832/2909630/Memento+ Fitoterapico/a80ec477-bb36-4ae0-b1d2-e2461217e06b>. Acesso em: 18 abr. 2018

19. BRASIL. Agência Nacional de Vigilância Sanitária(ANVISA). Resolução RDC $n^{\circ} 24$, de 14 de junho de 2010. Dispõe sobre o registro de medicamentos específicos. Diário Oficial [da] República Federativa do Brasil, Brasília, DF, 17 jun. 2011. Disponível em: < http://pesquisa. in.gov.br/imprensa/jsp /visualiza/index.jsp?jornal=1\&pagina $=79 \& d a$ ta $=17 / 06 / 2011>$. Acesso em: 17 maio 2018.

20. BRASIL. Agência Nacional de Vigilância Sanitária (ANVISA). Resolução RDC $n^{\circ}$ 97, de 01 de agosto de 2016. Altera a Resolução - RDC no 24, de 14 de junho de 2011. Diário Oficial [da] República Federativa do Brasil, Brasília, DF, 03 ago. 2016. Disponível em: < http://pesquisa. in.gov.br/imprensa/ jsp/visualiza/index.jsp?jornal=1\&pagina $=32 \& d a$ ta=03/08/2016>. Acesso em: 17 maio 2018.

21. COOPER, R. J. Over-the-counter medicine abuse - a review of the literature. J Subst. Use, Londres, v. 18, n. 2, p. 82-107, 2013.

22. BRASS, E. P.; LOFSTEDT, R.; RENN, O. A decision-analysis tool for benefit-risk assessment of nonprescription drugs. J. Clin. Pharmacol., New York City, v. 53, n. 5, p. 475-482, 2013.

23. SENA, L. C. S. et al. Excipientes farmacêuticos e seus riscos à saúde: uma revisão de literature. Rev. Bras. Farm. Hosp. Serv. Saúde, São Paulo, v. 5, n. 4, p. 25-34, out./dez. 2014.

24. SILVA, A.V. A. et al. Presença de excipientes com potencial para indução de reações adversas em medicamentos comercializados no Brasil. Rev. Bras. Cienc. Farm., São Paulo, v. 44, n. 3, p. 397-405, jul./set. 2008.

25. MONTALTO, M. et al. Low-dose lactose in drugs neither increases breath hydrogen excretion or causes gastrointestinal symptoms. Aliment. Pharmacol. Ther., Medford, v. 28, p. 1003-1312, 2008.

26. STEFANI, G. P. et al. Presença de corantes e lactose em medicamen- tos: avaliação de 181 produtos. Rev. Bras. Alergia Imunopatol., São Paulo, v. 32, n. 1, p. 18-26, 2009

27. ROLIM, L. A. et al. Aplicações de revestimento em formas farmacêuticas sólidas na indústria farmacêutica. Rev. Bras. Farm., Rio de Janeiro, v. 90, n. 3, p. 224-230, 2009.

28. CHASSANY, O.; MICHAUX, A.; BERGMANN, J. F. Drug-induced diarrhoea. Drug Saf., New Zealand, v. 22, n. 1, p. 53-72, jan. 2000.

29. EADALA, P. et al. Quantifying the 'hidden' lactose in drugs used for the treatment of gastrointestinal conditions. Aliment. Pharmacol. Ther. Medford, v. 29, n. 6, p. 677-687, Mar. 2009.

30. BEDINE, M. S.; BAYLESS, T. M. Intolerance of small amounts of lactose byind ividuals with low lactase levels. Gastroenterology, Henderson, v. 65, n. 5, p. 735-743, 1973.

31. SUAREZ, F. L.; SAVAIANO, D. A.; LEVITT, M. D. A comparison of symptoms after the consumption of milk or lactose-hydrolyzed milk by people with self-reported severe lactose intolerance. N. Engl. J. Med., Massachussets, v. 333, p. 1-4, 1995.

32. BRASIL. Agência Nacional de Vigilância Sanitária (ANVISA). Resolução $R D C n^{\circ} 135$, de 08 de fevereiro de 2017. Altera a Portaria SVS/MS no 29, de 13 de janeiro de 1998, que aprova o regulamento técnico referente a alimentos para fins especiais, para dispor sobre os alimentos para dietas com restrição de lactose. Diário Oficial [da] República Federativa do Brasil, Brasília, DF, 09 fev. 2017. Disponível em: < http://pesquisa. in.gov.br/imprensa/jsp/visualiza /index.jsp ?jornal=1\&pagina $=44 \& d a$ ta $=09 / 02 / 2017>$. Acesso em: 22 maio 2018.

33. BRASIL. Agência Nacional de Vigilância Sanitária (ANVISA). Resolução RDC $n^{\circ} 136$, de 08 de fevereiro de 2017. Estabelece os requisitos para declaração obrigatória da presença de lactose nos rótulos dos alimentos. Diário Oficial [da] República Federativa do Brasil, Brasília, DF, 09 fev. 2017. Disponível em: < http://pesquisa.in.gov.br/imprensa/jsp/ visualiza/index.jsp?jornal $=1 \&$ pagina $=44 \&$ data $=09 / 02 / 2017>$. Acesso em: 22 mai. 2018.

34. BANAS, A. et al. Is it possible to find presence of lactose in pharmaceuticals? - preliminary studies by ATR-FTIR spectroscopy and chemometrics. Spectrochim. Acta, A. Mol. Biomol. Spectrosc., Amsterdã, v. 171, p. 280-286, 2017.

35. CARVALHO, M. F. C. et al. Polifarmácia entre idosos do Município de São Paulo: Estudo SABE. Rev. Bras. Epidemiol., São Paulo, v. 15, n. 4, p. 817-827, 2012. DOI:10.1590/S1415-790X2012000400013.

36. PEREIRA, K. G. et al. Polifarmácia em idosos: um estudo de base populacional. Rev. Bras. Epidemiol., São Paulo, v. 20, n. 2, abr./jun. 2017. DOI:10.1590/1980-5497201700020013.

37. AGÊNCIA NACIONAL DE VIGILÂNCIA SANITÁRIA (ANVISA). Medicamentos genéricos: estatísticas e listas. Disponível em: < http:// portal. anvisa.gov.br/medicamentos-genericos-registrados>. Acesso em: 07 jun. 2018.

38. RAMOS, G.; MORAIS, D. C. M. de. Revisão de literatura sobre excipientes em farmácia de manipulação. Foco, Vila Velha, v. 4, n. 5, jul./dez. 2013.

39. PESSANHA, A. F. de V, et al. Influência dos excipientes multifuncionais no desempenho dos fármacos em formas farmacêuticas. Rev. Bras. Farm., Rio de janeiro, v. 93, n. 2, p. 136-45, 2012.
Submetido em: $19 / 11 / 2018$

Aceito em: 29/11/2018 


\section{DECLARAÇÃO DE RESPONSABILIDADE}

"Certificamos que o artigo enviado à Revista de Ciências Médicas e Biológicas é um trabalho original, sendo que o seu conteúdo não foi ou não está sendo considerado para publicação em outra revista, seja no formato impresso ou eletrônico."

Mttoparrientos Marcia Otto Barrientos

Dlixeiva

Fermanda Cristina Figueira Teixeira

Lowaudoe

Roberto Paulo Correia Araújọ 